\title{
ON UNIFORM ASYMPTOTIC STABILITY FOR NONLINEAR INTEGRO-DIFFERENTIAL EQUATIONS OF VOLTERRA TYPE
}

\author{
Natalia Sedova \\ Department of Mathematics, Information and Aviation Technologies \\ Ulyanovsk State University \\ Russia \\ sedovano@ulsu.ru
}

Article history:

Received 14.10.2019, Accepted 28.11.2019

\begin{abstract}
The specifics of the application of Razumikhin technique to the stability analysis of Volterra type integrodifferential equations are considered. The equation can be nonlinear and nonautonomous. We propose new sufficient conditions for uniform asymptotic stability of the zero solution using the phase space of a special construction and constraints on the right side of the equation. In the presented constraints we can analyze stability, relying not only on the behavior of the auxiliary function along the solutions, but also on the properties of the socalled limiting equations.
\end{abstract}

\section{Key words}

Nonlinear systems, integro-differential equations, nonautonomous, unbounded delay, LyapunovRazumikhin functions, limiting equations.

\section{Introduction}

Volterra integro-differential equations (IDE) are widely employed in modern mathematical modeling, including the description of control systems. In particular, IDE are used in modeling heat flows, processes in electrical engineering, hereditary mechanical systems (see, e.g., references in [Tunc and Tunc, 2018]); stabilization problems of mechanical systems with PI and PID regulators also can be reduced to a Volterra IDE [Andreev and Peregudova, 2017].

Since such models takes into account the history of the system, approaches that are suitable for equations with delay are applied to Volterra-type equations. In particular, the method of Lyapunov-Krasovskii functionals is used for stability analysis. In recent works using this method, it has been possible to increase the efficiency of this method by using the technique of limiting equations
[Andreev and Peregudova, 2018a; Andreev and Peregudova, 2018b]. A similar approach is applicable to the other branch of the direct method - the Razumikhin technique [Haddock and Terjéki, 1990; Hara et al., 1992; Seifert, 1974; Seifert, 1973]. However, constructions convenient for the use of functionals are, in this case, ineffective in applied problems. This paper proposes to use a special space with fading memory, in which, under fairly general assumptions, it is possible to construct limiting equations and constructively modify the classical Razumikhin conditions. This allows us to continue the development of a number of previously known results [Hino and Murakami, 1991; Seifert, 1973; Sedova, 2009].

\section{Preliminaries}

We use the following notation: $R^{n}$ is a real $n$ dimensional space equipped with the norm $|\cdot|, R^{+}=$ $[0,+\infty), R^{-}=(-\infty, 0], D=\left\{(t, s) \in R^{+} \times R: s \leqslant\right.$ $t\}$. The open $a$-neighborhood of zero and the closed one will be denoted by $R_{a}$ and $\bar{R}_{a}$ respectively.

We consider the equation

$$
\dot{x}(t)=G(t, x(t))+\int_{0}^{t} H(t, s, x(s)) d s,
$$

where $x(t) \in R^{n}, t \in R^{+}, G \in C\left(R^{+} \times R^{n}, R^{n}\right)$, $H \in C\left(D \times R^{n}, R^{n}\right)$.

Also, for a continuous function $x:(-\infty, A] \rightarrow R^{n}$, $0 \leqslant A \leqslant+\infty$, we define for each $t \leqslant A$ the function $x_{t}: R^{-} \rightarrow R^{n}$ by the formula $x_{t}(s)=x(t+s)$.

We assume that $G(t, 0)=0, H(t, s, 0)=0$, so (1) admits the zero solution.

The equation (1) can be interpreted as an equation with unbounded delay [Corduneanu and Lakshmikantham, 1980] with the delay function $r(t)=t$. When 
studying such equations in many studies a functional argument with a fixed $t \in R^{+}$is considered to be a continuous function defined on the interval $[-r(t), 0]$, with the norm $|\varphi|_{t}=\sup _{-r(t) \leqslant s \leqslant 0}|\varphi(s)|$ dependent on $t$ (see, for example, [Hara et al., 1992; So et al., 1996; Seifert, 1974; Seifert, 1973]).

Another option is to use as a phase space $B$ with fading memory, which is "traditional" for equations with infinite delay. To do this, we consider the right-hand side of the equation (1) as a functional $f(t, \varphi)$ defined for $t \in R^{+}$and $\varphi: R^{-} \rightarrow R^{n}$, although, in contrast to a general equation with unbounded delay, it is natural to consider a finite-dimensional vector $x^{0} \in R^{n}$ as a initial point. Defining the function $x_{t} \in B$ for a solution of (1), we assume that $x(t+s)=x(0)$ for $s \leqslant-t$.

Next, we use a separable space $B$ with uniformly fading memory, which we will call an admissible space [Atkinson and Haddock, 1988; Murakami and Naito, 1989; Hale and J, 1978]. Since definitions of such spaces differ in different publications, for specificity we refer to [Sedova, 2009]. The norm in space $B$ will be denoted by $|\cdot|_{B}$, and the sets $B_{h}=\left\{\varphi \in B:|\varphi|_{B}<h\right\}$, $\bar{B}_{h}=\left\{\varphi \in B:|\varphi|_{B} \leqslant h\right\}$ for $h>0$ will be used below.

For the convenience of the following formulations, we now introduce assumptions regarding the fundamental properties of (1).

\section{Assumption 1.}

1.1. For every initial point $x^{0} \in R^{n}$ there exists a noncontinuable solution $x(t)$ of (1), which is determined for $t \in[0, \beta)$ with some $\beta>0$, i.e., it is a continuous function satisfying (1) on $[0, \beta)$ with $x(0)=x^{0}$. In addition, for any $\varepsilon>0$ and $x(t)$, which is a noncontinuable solution of (1) on $[0, \beta)$ with $\left|x^{0}\right|<\varepsilon$, we have either $\beta=+\infty$ or $\left|x_{t_{1}}\right|_{B}=\varepsilon$ for some $t_{1} \in(0, \beta)$.

In addition, if the Lipschitz condition is satisfied, namely, for any $t \in R^{+}, \varphi, \psi \in B_{h}$ the estimate

$$
\left|f\left(t, \varphi_{2}\right)-f\left(t, \varphi_{1}\right)\right| \leqslant l(h)\left|\varphi_{2}-\varphi_{1}\right|_{B}
$$

is valid for some $l(h)>0$, then such a solution is unique and continuously depends on initial point.

1.2. If $x\left(t, \varphi_{0}\right)$ is a bounded solution of (1) which is determined for all $t \geqslant 0$, then the positive orbit $\left\{x_{t}\left(x^{0}\right)\right.$ : $t \geqslant 0\}$ is precompact in $B$, and if $x\left(t_{n}+s\right) \rightarrow \varphi(s)$ as $n \rightarrow \infty$ uniformly in $s \in[-T, 0]$ for any $T>0$ then $\varphi \in B$ and $\left|x_{t_{n}}-\varphi\right|_{B} \rightarrow 0$ as $n \rightarrow \infty$.

Note that, in contrast to the case of an equation with a finite delay, the fulfillment of Assumption 1 depends not only on the right side of the equation, but also on the choice of the phase space.

The "axiomatic" definition of phase space has shown its high efficiency in the study of equations with an infinite delay, primarily in questions of asymptotic behavior and stability. The properties defined by "axioms" ensure that the Assumption 1 is valid for the right-hand side satisfying fairly weak restrictions [Hale and J, 1978].
As an admissible space for (1) we choose a subspace of space $C_{g}$ [Haddock and Zhao, 2006; Murakami and Naito, 1989]. This space consists of continuous functions $\varphi: R^{-} \rightarrow R^{n}$ such that $\sup _{s \leqslant 0}|\varphi(s)| / g(s)<\infty$ with the function $g$ satisfying the following conditions: $g \in C\left(R^{-},[1, \infty)\right)$ is a nonincreasing function, $g(0)=$ $1, \lim _{u \rightarrow 0-}[g(s+u) / g(s)] \rightarrow 1$ uniformly on $R^{-}$, $g(s) \rightarrow \infty$ as $s \rightarrow-\infty$. In applications, $e^{-\gamma s}$ with $\gamma>0$ is often used as $g$.

We now discuss the conditions on the right-hand side $f(t, \varphi)$ of (1) that enforce the following assumption.

\section{Assumption 2.}

2.1. For every $a>0$ there exists $c=c(a)$ such that $|f(t, \varphi)| \leqslant c(a)$ for all $(t, \varphi) \in R^{+} \times \bar{B}_{a}$.

2.2. The functional $f(t, \varphi)$ is uniformly continuous on every set of the form $R^{+} \times K$ with a compact $K \subset B$, i.e. for any compact $K \subset B$ and for any small $\varepsilon>0$ there is a $\delta=\delta(\varepsilon, K)>0$ such that the conditions $\left(t_{1}, \varphi_{1}\right),\left(t_{2}, \varphi_{2}\right) \in R^{+} \times K,\left|t_{1}-t_{2}\right|<\delta$, and $\mid \varphi_{1}-$ $\left.\varphi_{2}\right|_{B}<\delta$ imply $\left|f\left(t_{1}, \varphi_{1}\right)-f\left(t_{2}, \varphi_{2}\right)\right|<\varepsilon$.

It is known that if $B$ is an admissible fading memory space then Assumption 2 imply Assumption 1 [Hale and J, 1978].

In addition, Assumption 2 ensures the existence of limiting functionals for the right-hand side of the equation in the topology of uniform convergence on compact sets. The latter property makes it possible to use the technique of limiting equations to analyze the original equation [Andreev, 2005; Druzhinina and Sedova, 2014; Sedova, 2008].

Now we introduce the following assumption regarding the functions $G$ and $H$ in the right side of the equation (1).

\section{Assumption 3.}

3.1. For any $a, k>0$ there exist $L_{0}(a)$ and $L_{1}(a, k)$ such that $|G(t, x)-G(t, y)| \leqslant L_{0}(a)|x-y|, \mid H(t, t+$ $s, x)-H(t, t+s, y)\left|\leqslant L_{1}(a, k)\right| x-y \mid$ for all $(t, s, x),(t, s, y) \in R^{+} \times[-k, 0] \times \bar{R}_{a}$.

3.2. For any $\varepsilon>0$ and $a>0$ there exists $S>0$ such that $\int_{-\infty}^{-S}\left|H\left(t, t+s, \varphi_{1}(s)\right)-H\left(t, t+s, \varphi_{2}(s)\right)\right| d s<$ $\varepsilon\left|\varphi_{1}-\varphi_{2}\right|_{B C}$ for all $t \in R^{+}, \varphi_{1}, \varphi_{2} \in \overline{B C}_{a}=\{\varphi \in$ $\left.C\left(R^{-}, R^{n}\right):|\varphi|_{B C}=\sup _{s \leqslant 0}|\varphi(s)| \leqslant a\right\}$.

3.3. $G(t, x)$ is uniformly continuous on every set of the form $R^{+} \times \bar{R}_{a}$; for all $\varepsilon, a, k>0$ there exists $\delta(\varepsilon, a, k)>$ 0 such that $\left|H\left(t_{1}, t_{1}+s, x\right)-H\left(t_{2}, t_{2}+s, x\right)\right|<\varepsilon$ for all $t_{1}, t_{2} \in R^{+}:\left|t_{1}-t_{2}\right|<\delta, x \in \bar{R}_{a}, s \in[-k, 0]$.

Assumption 3 is a generalization of the assumptions from [Hino and Murakami, 1991] where precompact conditions for the linear Volterra IDE with an almost periodic right-hand side are considered. The conditions of Assumption 3 also differ from those from the papers [Andreev and Peregudova, 2018a; Andreev and Peregudova, 2018b; Andreev and Peregudova, 2017] offering precompact results for a non-periodic right-hand side. 


\section{Volterra IDE as an Unbounded Delay Equation in a Fading Memory Space: Stability Results}

The following statement is proved.

\section{Theorem 1.}

If the conditions of Assumption 3 are satisfied, then the right-hand side of (1) satisfies Assumption 2 with $B$ being an admissible subspace of $C_{g}$ for some appropriate function $g$.

Proof. We will prove that there exists a function $g$ that meets the conditions from section 2 and such that the functional $f_{0}(t, \varphi)=\int_{-t}^{0} H(t, t+s, \varphi(s)) d s$ is uniformly continuous and bounded on a set $R^{+} \times\left(\bar{C}_{g}\right)_{h}=$ $R^{+} \times\left\{\varphi \in C_{g}:|\varphi|_{g} \leqslant h\right\}$ for any $h>0$. This will be sufficient to prove the theorem.

First we show that for any $h>0$ there is a $\beta(h)>0$ such that $\int_{-\infty}^{0}|H(t, t+s, \varphi(s))| d s \leqslant \beta(h)$ for all $t \in R^{+}, \varphi \in\left(\bar{C}_{g}\right)_{h}$. This will mean the required boundedness.

We set $\varepsilon_{n}=1 / n^{2}$ and fix arbitrary $\alpha_{n}>0$ with $\alpha_{n} \rightarrow$ $+\infty$ being a strictly increasing sequence. Assumption 3.2 implies that for every $n$ there exists $k_{n}>0$ such that $k_{n+1}>k_{n}, k_{n} \rightarrow+\infty$ as $n \rightarrow \infty$, and $\int_{-\infty}^{-k_{n}} \mid H(t, t+$ $s, \varphi(s)) \mid d s<\varepsilon_{n}$ for all $t \in R^{+}, \varphi \in \overline{B C}_{\alpha_{n}(2+n)}$. We define the function $g \in C\left(R^{-},[1,+\infty)\right)$ as follows: $g\left(-k_{n}\right)=1+n, g$ is linear on $\left[-k_{1}, 0\right]$ and on $\left[-k_{n+1},-k_{n}\right]$ for any $n \geqslant 1$.

Put an arbitrary $h>0$ and $\varphi \in\left(\bar{C}_{g}\right)_{h}$. Notice that $h \leqslant \alpha_{n}$ for all $n \geqslant N=N(h)>0$. For every $n$ define a function

$$
\varphi_{n}(s)=\left\{\begin{aligned}
\varphi\left(-k_{n}\right), s & \in\left[-k_{n}, 0\right] ; \\
\varphi(s), s & \in\left[-k_{n+1},-k_{n}\right] \\
\varphi\left(-k_{n+1}\right), & s \in\left(-\infty,-k_{n+1}\right] .
\end{aligned}\right.
$$

Then $\left|\varphi_{n}(s)\right| \leqslant h g\left(-k_{n+1}\right)=h(2+n)$ for all $s \in R^{-}$, therefore $\varphi_{n} \in \overline{B C}_{h(2+n)}$. So, for any $t \in R^{+}$we have $\int_{-\infty}^{0}|H(t, t+s, \varphi(s))| d s=$ $\int_{-k_{1}}^{0}|H(t, t+s, \varphi(s))| d s+\sum_{n=1}^{N} \int_{-k_{n+1}}^{-k_{n}} \mid H(t, t+$ $s, \varphi(s))\left|d s+\sum_{n=N+1}^{\infty} \int_{-k_{n+1}}^{-k_{n}}\right| H(t, t+s, \varphi(s)) \mid d s \leqslant$ $\int_{-k_{1}}^{0}|H(t, t+s, \varphi(s))| d s+\sum_{n=1}^{N} \int_{-k_{n+1}}^{-k_{n}} \mid H(t, t+$ $\left.s, \varphi_{n}(s)\right)\left|d s+\sum_{n=N+1}^{\infty} \int_{-\infty}^{-k_{n}}\right| H\left(t, t+s, \varphi_{n}(s)\right) \mid d s \leqslant$ $C+\sum_{n=N+1}^{\infty}\left(1 / n^{2}\right)$ with $C$ being a constant which depends on $N, k_{1}, k_{N}$, and $h$ (see Assumption 3.1). Because of this, for the function $g$ and for every $h>0$ there is $\beta=\beta(h)>0$ that provides an estimate $\int_{-\infty}^{0}|H(t, t+s, \varphi(s))| d s \leqslant \beta$ for all $t \in R^{+}$and $\varphi \in\left(\bar{C}_{g}\right)_{h}$.

Now we choose $j \geqslant N$ satisfying the conditions $\sum_{n=j}^{\infty}\left(1 / n^{2}\right)<\varepsilon / 6$. Then $k_{0}>k_{j}$ provides $\int_{-\infty}^{-k_{0}}|H(t, t+s, \varphi(s))| d s \leqslant \sum_{n=j}^{\infty} \int_{-k_{n+1}}^{-k_{n}} \mid H(t, t+$ $s, \varphi(s))\left|d s \leqslant \sum_{n=j}^{\infty} \int_{-\infty}^{-k_{n}}\right| H\left(t, t+s, \varphi_{n}(s)\right) \mid d s \leqslant$ $\sum_{n=j}^{\infty}\left(1 / n^{2}\right)<\varepsilon / 6$ for all $t \in R^{+}$and $\varphi \in\left(\bar{C}_{g}\right)_{h}$.

Next we prove that $f_{1}(t, \varphi)=\int_{-\infty}^{0} H(t, t+s, \varphi(s)) d s$ is uniformly continuous and bounded on a set
$R^{+} \times\left(\bar{C}_{g}\right)_{h}$ for any $h>0$. Assumption 3.3 with $\delta_{1}=\delta\left(\varepsilon /\left(6 k_{0}\right), h g\left(-k_{0}\right), k_{0}\right)$ yields $\mid \int_{-k_{0}}^{0}\left(H\left(t_{1}, t_{1}+s, \varphi(s)\right)-H\left(t_{2}, t_{2}+s, \varphi(s)\right) d s \mid<\right.$ $\varepsilon / 6$ for $t_{1}, t_{2} \in R^{+}$with $\left|t_{1}-t_{2}\right|<\delta_{1}$ and $\varphi \in\left(\bar{C}_{g}\right)_{h}$. It follows that $\left|f_{1}\left(t_{1}, \varphi\right)-f_{1}\left(t_{2}, \varphi\right)\right| \leqslant$ $\left|\int_{-k_{0}}^{0}\left(H\left(t_{1}, t_{1}+s, \varphi(s)\right)-H\left(t_{2}, t_{2}+s, \varphi(s)\right)\right) d s\right|+$ $\int_{-\infty}^{-k_{0}}\left|H\left(t_{1}, t_{1}+s, \varphi(s)\right)\right| d s+\int_{-\infty}^{-k_{0}} \mid H\left(t_{2}, t_{2}+\right.$ $s, \varphi(s)) \mid d s<\varepsilon / 2$. At last, the following estimate is true: $\left|f_{1}\left(t_{1}, \varphi_{1}\right)-f_{1}\left(t_{2}, \varphi_{2}\right)\right| \leqslant$ $\left|f_{1}\left(t_{1}, \varphi_{1}\right)-f_{1}\left(t_{2}, \varphi_{1}\right)\right|+\mid f_{1}\left(t_{2}, \varphi_{1}\right)-$ $f_{1}\left(t_{2}, \varphi_{2}\right)|\leqslant| f_{1}\left(t_{1}, \varphi_{1}\right)-f_{1}\left(t_{2}, \varphi_{1}\right) \mid+$ $\left|\int_{-k_{0}}^{0}\left(H\left(t_{2}, t_{2}+s, \varphi_{1}(s)\right)-H\left(t_{2}, t_{2}+s, \varphi_{2}(s)\right)\right) d s\right|+$ $\int_{-\infty}^{-k_{0}}\left|H\left(t_{2}, t_{2}+s, \varphi_{1}(s)\right)\right| d s+\int_{-\infty}^{-k_{0}} \mid H\left(t_{2}, t_{2}+\right.$ $\left.s, \varphi_{2}(s)\right) \mid d s$. We can assume that the second term in this sum is less than $\varepsilon / 6$ for sufficiently small $\left|\varphi_{1}-\varphi_{2}\right|_{g}$, since on the compact $\bar{R}_{h g\left(-k_{0}\right)}$ the function $H(t, t+s, x)$ is uniformly continuous in $x$. As the result, $\left|f_{1}\left(t_{1}, \varphi_{1}\right)-f_{1}\left(t_{2}, \varphi_{2}\right)\right|<\varepsilon$ for $t_{1}, t_{2} \in R^{+}:\left|t_{1}-t_{2}\right|<\delta_{0}, \varphi_{1}, \varphi_{2} \in\left(\bar{C}_{g}\right)_{h}$ : $\left|\varphi_{1}-\varphi_{2}\right|_{g}<\delta_{0}$ where $\delta_{0}=\delta_{0}(\varepsilon, h)>0$ is sufficiently small.

Finally, we prove the uniform continuity of the functional $f_{0}$.

Choose $t_{1}, t_{2} \in R^{+}, t_{2}<t_{1}<t_{2}+\delta$, $\varphi_{1}, \varphi_{2} \in\left(C_{g}\right)_{h}$. Consider the difference $\mid f_{0}\left(t_{1}, \varphi_{1}\right)-$ $f_{0}\left(t_{2}, \varphi_{2}\right)\left|\leqslant \int_{-t_{2}}^{0}\right| H\left(t_{1}, t_{1}+s, \varphi_{1}(s)\right)-H\left(t_{2}, t_{2}+\right.$ $\left.s, \varphi_{2}(s)\right)|d s+| \int_{-t_{1}}^{-t_{2}} H\left(t_{1}, t_{1}+s, \varphi_{1}(s)\right) d s \mid$. The first term in the estimate does not exceed $\mid f_{1}\left(t_{1}, \varphi_{1}\right)$ $f_{1}\left(t_{2}, \varphi_{2}\right) \mid$ and according to the above it is less than $\varepsilon / 2$, provided that $\left|\varphi_{1}-\varphi_{2}\right|_{g}<\delta$ with $\delta \leqslant \delta_{0}(\varepsilon / 2, h)$.

Then, sequentially considering cases $t_{1} \leqslant k_{0}, t_{2} \geqslant k_{0}$, and $t_{2}<k_{0}<t_{1}$ and using Assumption 3.1 and the above estimate of the integral $\int_{-\infty}^{-k_{0}}|H(t, t+s, \varphi(s))| d s$, we obtain that for a sufficiently small $\delta$ the inequality $\left|\int_{-t_{1}}^{-t_{2}} H\left(t_{1}, t_{1}+s, \varphi_{1}(s)\right) d s\right|<\varepsilon / 2$ holds.

Because of this, $\left|f_{0}\left(t_{1}, \varphi_{1}\right)-f_{0}\left(t_{2}, \varphi_{2}\right)\right|<\varepsilon$ subject to $\left|t_{1}-t_{2}\right|<\delta,\left|\varphi_{1}-\varphi_{2}\right|_{g}<\delta, \varphi_{1}, \varphi_{2} \in\left(\bar{C}_{g}\right)_{h}$.

So the proof is complete.

Thus, under Assumption 3 the fundamental properties of the solutions (Assumption 1) hold and it is possible to construct equations that are limiting ones for (1).

If the sequence $\left\{G\left(t+t_{k}, x\right)\right\}$ converges to $G^{*}(t, x)$, and the sequence $\left\{H\left(t+t_{k}, t+t_{k}+s, x\right)\right\}$ converges to $H^{*}(t, t+s, x)$ as $t_{k} \rightarrow+\infty$ uniformly on the corresponding compact subsets then a limiting equation for (1) has the form:

$$
\dot{x}(t)=G^{*}(t, x(t))+\int_{-\infty}^{t} H^{*}(t, s, x(s)) d s .
$$

It is clear that equation (2) can be considered in the same phase space as the original one, and the limiting functions satisfy the same constraints as the original ones. 
In many practical problems, it is natural to assume that the solutions belong to the space $B C$ of bounded continuous functions $\varphi: R^{-} \rightarrow R$ with the norm $|\varphi|_{B C}$. Most of the studies on the stability of equations with unbounded delay use exactly this space (see, for example, [Hino and Murakami, 1991; Murakami, 1985; Rao and Sivasundaram, 1988; Parrot, 1981; Parrot, 1982]).

Note that if we take the space $B C$ as the phase space, then the fundamental properties of solutions (Assumption 1) generally may not take place even under rather restrictive conditions on the right-hand side of the equation (see, for example, [Seifert, 1982]). The axiomatic definition of an admissible space is convenient in constructing the theory and does not cause problems in applications, since functions from the space $B C$ belong to any admissible space $B$. However, for a Volterra-type equation, the use of $B C$ space does not create such problems. Such a simplification is possible, including due to the structure of the right side of the system. A significant advantage is the fact that the structure of the functional space used for constructing limiting functionals is simplified compared to the known constructions for the infinite delay case [Andreev and Peregudova, 2018a; Andreev and Peregudova, 2018b].

However, from the point of view of stability analysis, additional specifics arise here. The dependence of the right-hand side of the equation on the history leads to the fact that even in the case of a linear equation, it is usually possible to obtain only sufficient stability conditions via the direct method.

The use of Lyapunov-Krasovskii functionals was considered in [Andreev and Peregudova, 2018a; Andreev and Peregudova, 2018b; Burton, 1982; Burton, 1980] and others. In this case, the use of the phase space $B C$ turns out to be natural and convenient from the point of view of presentation and verification of stability conditions.

In some cases, the use of the functional turns out to be unnecessarily difficult, while sufficient conditions in terms of the ordinary Lyapunov function (with an additional Razumikhin-type condition) can be easily verifiable and sometimes quite close to necessary. But then the question arises about the correct modification of the Razumikhin condition (the original one is applicable to systems with bounded delay). As with the point of view of the fundamental properties of the solutions (1), the choice of phase space is also important from the standpoint of stability studies. By virtue of the choice of the space $B C$, it seems a natural "immediate" generalization of the Razumikhin condition, that is, a change of the interval over which the history is taken into account when estimating the derivative. However, this approach is ineffective; in particular, the sign-definiteness of the derivative $V^{\prime}$ on the set of functions defined by the inequality $V(s, \psi(s))<\eta(V(t, \psi(t))), s \in\left[t_{0}, t\right]$ ( $\eta$ is a K-class function with $\eta(u)>u$ for $u>0$ ), does not guarantee asymptotic stability. This was noted, in particular, in [Seifert, 1974; Seifert, 1973], where alternative
Razumikhin-type conditions for Volterra-type equations were proposed. Examples of linear equations with unbounded delay from [Driver, 1962] are also illustrative.

Possible solutions to this problem are proposed for example in [Murakami, 1985; Seifert, 1974; Seifert, 1973; Parrot, 1981]. In [Murakami, 1985] the right-hand side of the original equation is represented as a sum of a functional depending on $x(t+s), s \in[-r, 0]$ (term with finite delay), and a "tail", which is treated as a perturbation. Based on the theorems on uniform stability and uniform asymptotic stability for perturbed equations with finite delay, results in terms of Lyapunov-Razumikhin functions are obtained in [Murakami, 1985] for equations with infinite delay. In [Seifert, 1974; Seifert, 1973] the derivative is estimated under the condition $V(s, x(s))<$ $\eta(V(t, x(t)))$ for all $s \in\left[t_{0}, t\right]$ where $t_{0}=\max \{0, t-r\}$ with some $r>0$, and in [Parrot, 1981] that condition take the form $\sup _{s \leqslant 0} e^{\gamma s} V(t+s, x(t+s))=V(t, x(t))$ $(\gamma>0)$.

Consideration of (1) in a space with fading memory allows, in particular, applying to that equation results on asymptotic stability and instability for equations with infinite delay in an admissible function space. In some cases this approach leads to less restrictive parametric conditions. Given the selected space, for stability analysis of the zero solution of (1) it is convenient to use the Lyapunov-Razumikhin pair [Haddock and Terjéki, 1990] with a positive definite function $V(x)$ and the functional $W(\varphi)=\sup _{s \leqslant 0} V(\varphi(s) / g(s))$. So, we get the following result.

\section{Theorem 2.}

Suppose that there exists a positive definite continuously differentiable function $V(x)$ such that one of the following conditions is satisfied:

1. the derivative of $V(x)$ by virtue of (1) is negative definite for $\varphi \in C_{g}$ satisfying $\sup _{s \leqslant 0} V(\varphi(s) / g(s)) \leqslant V(\varphi(0))$ and for sufficiently large $t$.

2. the derivative $\frac{d}{d t} V(x(t))$ of $V(x)$ by virtue of every limiting equation (2) does not exceed a function $W(x(t))$ for $\varphi \in C_{g}$ satisfying $\sup _{s \leqslant 0} V(\varphi(s) / g(s)) \leqslant V(\varphi(0))$, and $W(x)$ is negative definite.

Then the zero solution of the equation (1) is uniformly asymptotically stable.

Other results can be obtained in terms of Lyapunov functions from sufficient asymptotic stability conditions for equations with infinite delay, including statements in terms of functions with a semidefinite estimate of the derivative or semidefinite functions [Sedova, 2008].

Here, we give one such result in terms of the function $V=V(x)$.

Some additional notation is needed. First, let $T>0$ be such that $g(s)>1$ for all $s \leqslant-T$ (the existence of such $T$ follows from the properties of the function $g$, see [Sedova, 2009]). Second, define for given functions $V(x)$ 
and $W(t, x)$ and for any $T, c>0$ the set $K_{V W}(T, c)$ consisting of solutions $x^{*}(t)$ of limiting equations (2) with the following properties: at any interval of length $T$ there is $t$ such that $\max _{-T \leqslant s \leqslant 0} V\left(x^{*}(t+s)\right)=$ $V\left(x^{*}(t)\right)=c$ and $W^{*}\left(t, x^{*}(t)\right)=0$ (here, the limiting equation with the solution $x^{*}(t)$ and the limiting function $W^{*}$ correspond to the same sequence $t_{k} \rightarrow+\infty$ ).

In the above notation, the following theorem is valid.

\section{Theorem 3.}

Suppose that there exists a positive definite continuously differentiable function $V(x)$ such that the derivative of $V(x)$ by virtue of (1) meets the condition $\frac{d}{d t} V(x(t)) \leqslant W(t, x(t)) \leqslant 0$ for $\varphi \in C_{g}$ satisfying $\sup _{s \leqslant 0} V(\varphi(s) / g(s)) \leqslant V(\varphi(0))$ and for sufficiently large values of $t$.

If, in addition, for any sufficiently small $c>0$ there is $T(c) \geqslant T$ such that the set $K_{V W}(T(c), c)$ is empty, then the zero solution of (1) is uniformly asymptotically stable.

In view of Theorem 1, the proof of Theorem 3 and some other results on asymptotic stability for equation (1) in terms of Lyapunov functions can be obtained directly from the results of [Sedova, 2008].

\section{Examples}

Note that the proof of Theorem 1 remains valid for any function $g(s)$ meeting the inequality $\int_{-\infty}^{-k_{n}} \mid H(t, t+$ $s, \varphi(s)) \mid d s<\varepsilon_{n}$ for each positive integer $n, t \in R^{+}$, and $\varphi \in \overline{B C}_{\alpha_{n} g\left(-k_{n+1}\right)}$, whenever $k_{1} \geqslant r, k_{n+1}>k_{n}$, $k_{n} \rightarrow+\infty, \alpha_{n} \rightarrow+\infty$ as $n \rightarrow \infty$, and the series $\sum_{n=1}^{\infty} \varepsilon_{n}$ converges. Therefore, in specific examples, instead of the proposed piecewise linear function, another suitable one can be used.

Example 1. Consider a scalar equation

$$
\dot{x}(t)=-h(t) x(t)-b(t) x^{3}(t)+\int_{0}^{t} c(a t-s) x(s) d s,
$$

where $c \in L_{1}[0,+\infty), a>1 ; h(t) \geqslant 0$ and $b(t) \geqslant b_{0}>$ 0 are continuous functions. In [Burton and Somolinos, 1999] the following conditions for uniform asymptotic stability are obtained via Lyapunov-Krasovskii functional:

$$
\begin{gathered}
2 h(t) \geqslant[1+(1 / a)] \int_{(a-1) t}^{\infty}|c(v)| d v, \\
\int_{t}^{\infty}|c(u)| d u \in L_{1}[0,+\infty) .
\end{gathered}
$$

We denote $M(t)=\int_{-\infty}^{0}|c((a-1) t-s)| d s=$ $\int_{(a-1) t}^{\infty}|c(v)| d v$ (this function is bounded), then for an arbitrarily small $\delta>0$ there exists an appropriate function $g$ such that $\int_{-\infty}^{0}|c((a-1) t-s)| g(s) d s<M(t)+\delta$. Now using the function $V(x)=x^{2}$ and Theorem 2 we obtain that the zero solution (3) is uniformly asymptotically stable if the functions $h(t)$ and $b(t)$ are bounded and meet the inequality

$$
h(t) \geqslant \int_{(a-1) t}^{\infty}|c(v)| d v+\varepsilon
$$

for some $\varepsilon>0$.

Example 2. Consider a scalar equation

$$
\begin{gathered}
\dot{x}(t)=-(a(t)+b(t, x(t))) x(t)+ \\
\int_{0}^{t} m r(t, s, x(s)) e^{-d(t-s)} x(s) d s,
\end{gathered}
$$

where $a(t) \geqslant a, d>0,|r(t, s, y)| \leqslant a, m>0$ and Assumption 3 is valid. We also assume that the only limiting function for $b(t, x)$ is the zero function. In [Burton, 1980] the case $a(t)=1, b(t, x)=0, r=r(t, s)$ is considered and, based on fairly complex Lyapunov functionals, it is proved that the zero solution of the equation is asymptotically stable if there are constant $\beta \geqslant 1, \mu \geqslant 0$, such that $\alpha=\frac{m \beta}{d}+\sqrt{2} \mu \leqslant 1, \alpha-\mu<1$.

Using the results of [Murakami, 1985; Seifert, 1974; Seifert, 1973; Parrot, 1981] for the function $V(x)=x^{2}$ does not allow one to obtain a sign-definite estimate for the derivative.

Now, note that if $\frac{m}{d}<1$, then for some $\gamma>0$ we have $0<\frac{m}{d-\gamma}<1$. Fix such $\gamma$ and consider the equation in the space $C_{g}$ with the function $g(s)=e^{-\gamma s}$. Then, using Theorem 2 and the properties of the limiting equations [Druzhinina and Sedova, 2014], it is easy to prove the uniform asymptotic stability of the zero solution.

Example 3. Consider a scalar equation [Tunc and Tunc, 2018]:

$$
\begin{array}{r}
\dot{x}(t)=-p(t) x(t)+q(t, x(t))+ \\
\int_{0}^{t} C(t-s) h(x(s)) d s,
\end{array}
$$

where

$$
\begin{array}{r}
h(0)=0,|h(x)| \leqslant \gamma|x| \text { for } x \neq 0 ; \\
p(t)-\gamma \int_{t}^{\infty}|C(u-t)| d u>\delta>0 \text { for } t \in R^{+} ; \\
|q(t, x)| \leqslant r(t)|x| \text { for some } r(t) \in L_{1} .
\end{array}
$$

In [Tunc and Tunc, 2018] by using a Lyapunov functional, it is proved that under the conditions (4) the zero solution of the equation is asymptotically stable.

The inequality $p(t)-\gamma \int_{t}^{\infty}|C(u-t)| d u>\delta>0$ for $t \in R^{+}$implies $\int_{-\infty}^{0}|C(-s)| d s<\varepsilon=\left(\inf _{t \in R^{+}} p(t)-\right.$ $\left.\delta_{1}\right) / \gamma$ for some $\delta_{1} \in(0, \delta)$. Then [Atkinson and Haddock, 1988] there exists an appropriate function $g$ such that $\int_{-\infty}^{0}|C(-s)| g(s) d s<\varepsilon$. Using the function $V(x)=x^{2}$, we now obtain that $V^{\prime}$ is negative definite for $\varphi \in C_{g}$ satisfying $\sup _{s \leqslant 0} V(\varphi(s) / g(s)) \leqslant V(\varphi(0))$ and for sufficiently large $t$.

Then Theorem 2 implies the uniform asymptotic stability of the zero solution. 


\section{Conclusion}

For a non-linear Volterra-type IDE, Razumikhin conditions are modified based on the use of a special admissible phase space. The considered equation is represented as an equation with unbounded delay in an admissible space with uniformly fading memory. Proposed conditions for the right-hand side the equations provide the correctness of using such a space, as well as the possibility of constructing a family of limiting equations. Razumikhin-type theorems with modified conditions, combined with the properties of limiting equations, prove to be an effective means of analyzing the stability of the trivial solution of the equation under consideration with a wider scope of application than previously known ones.

\section{Acknowledgments}

This work was supported by Russian Foundation for Basic Research, project no.18-41-730022.

\section{References}

Andreev, A. (2005). Stability of nonautonomous functional differential equations. Ulyanovsk University Press, Ulyanovsk, Russia. in Russian.

Andreev, A. S. and Peregudova, O. (2017). Non-linear PI and PID regulators in mechanical system control. In 8th International Conference on Physics and Control (PhysCon 2017), vol. 53, pp. 22-38.

Andreev, A. S. and Peregudova, O. (2018a). Nonlinear regulators in position stabilization problem of holonomic mechanical system. Mechanics of Solids, 53 (7), pp. 22-38.

Andreev, A. S. and Peregudova, O. (2018b). On the stability and stabilization problems of Volterra integrodifferential equations. Russian Journal of Nonlinear Dynamics, 14 (3), pp. 387-407.

Atkinson, F. and Haddock, J. (1988). On determining phase spaces for functional differential equations. Funkcialaj Ekvacioj, 31, pp. 331-348.

Burton, T. (1980). Uniform stabilities for Volterra equations. J. of Differential equations, 36, pp. 40-53.

Burton, T. (1982). Construction of Lyapunov functionals for Volterra equations. J. Math. Anal. and Appl., 85, pp. 90-105.

Burton, T. and Somolinos, A. (1999). Asymptotic stability in differential equations with unbounded delay. EJQTDE, (13).

Corduneanu, C. and Lakshmikantham, V. (1980). Equations with unbounded delay: a survey. Nonlinear Analysis: Theory, Methods and Applications, 4, pp. 831877.

Driver, R. (1962). Existence and stability of solutions of a delay-differential system. Arch. Ration. Mech. and Anal., 10, pp. 401-426.

Druzhinina, O. and Sedova, N. (2014). Method of limiting equations for the stability analysis of equations with infinite delay in the Caratheodory conditions: II. Diff. Equat., 50, pp. 711-721.
Haddock, J. and Terjéki, J. (1990). On the location of positive limit sets for autonomous functional differential equations with infinite delay. J. Differential equations, 86, pp. 1-32.

Haddock, J. and Zhao, J. (2006). Instability for functional differential equations. Math. Nachr., 279, pp. 1491-1504.

Hale, J. and J, K. (1978). Phase space for retarded equations with infinite delay. Funkcialai Ekvacioj, 21, pp. 11-41.

Hara, T., Yoneyama, T., and Miyazaki, R. (1992). Some refinements of Razumikhin's method and their applications. Funkcialaj Ekvacioj, 35, pp. 279-305.

Hino, Y. and Murakami, S. (1991). Stability properties of linear Volterra equations. J. Differential equations, 89, pp. 121-137.

Murakami, S. (1985). Stability in functional differential equations with infinite delay. Tohoku Math. J., 36, pp. 561-570.

Murakami, S. and Naito, T. (1989). Fading memory spaces and stability prorerties for functional differential equations with infinite delay. Funkcialaj Ekvasioj, 32, pp. 91-105.

Parrot, M. (1981). Convergence of solutions of infinite delay differential equations with an underlying space of continuous functions. Lecture Notes in Mathematics, 846.

Parrot, M. (1982). The limiting behavior of solutions of infinite delay differential equations. J. of Math. Anal. and Appl., 87, pp. 603-627.

Rao, M. and Sivasundaram, S. (1988). Asymptotic stability for equations with unbounded delay. J. of Math. Anal. and Appl., 131, pp. 97-105.

Sedova, N. (2008). Development of the direct Lyapunov method for functional-differential equations with infinite delay. Mathematical Notes, 84 (6), pp. 826-841.

Sedova, N. (2009). Stability in systems with unbounded aftereffect. Automation and Remote Control, 70(9), pp. 1553-1564.

Seifert, G. (1973). Liapunov-Razumikhin conditions for stability and boundedness of functional differential equations of Volterra type. J. Differential equations, 14, pp. 424-430.

Seifert, G. (1974). Liapunov-Razumikhin conditions for asymptotic stability in functional differential equations of Volterra type. J. Differential equations, 16, pp. 289297.

Seifert, G. (1982). On Caratheodory conditions for functional differential equations with infinite delays. Rocky Mountain J. of Mathematics, 12 (4), pp. 615-619.

So, J.-H., Yu, J., and Chen, M.-P. (1996). Asymptotic stability for scalar delay differential equations. Funkcialaj Ekvacioj, 39, pp. 1-17.

Tunc, C. and Tunc, Q. (2018). New qualitative criteria for solutions of Volterra integro-differential equations. Arab Journal of Basic and Applied Sciences. 